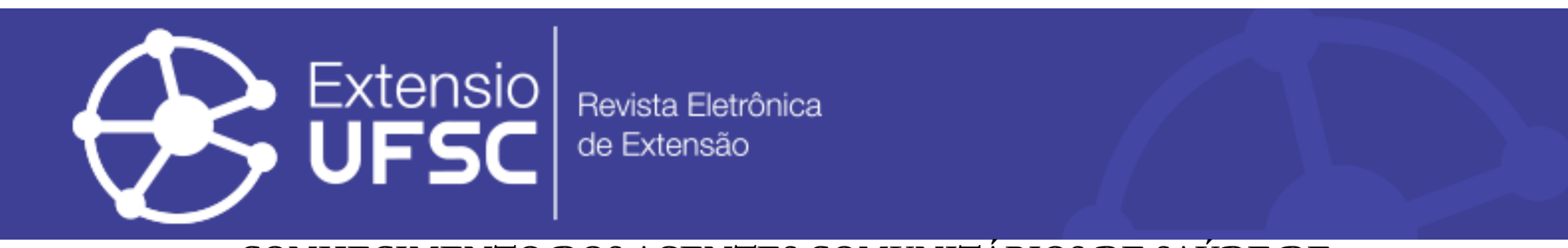

\title{
CONHECIMENTO DOS AGENTES COMUNITÁRIOS DE SAÚDE DE UM MUNICÍPIO BAIANO SOBRE PLANTAS MEDICINAIS
}

\author{
Bruno Rodrigues Alencar \\ Universidade Estadual de Feira de Santana \\ alencarbruno@yahoo.com.br \\ Eideclara Correia dos Santos \\ Hospitalar da União Médica Planos de Saúde S.A. \\ clara_ccds@hotmail.com
}

\author{
Gizelly Braga Pires \\ Universidade Estadual de Feira de Santana \\ gizellyp@yahoo.com.br \\ Tatiane de Oliveira Silva Alencar \\ Universidade Estadual de Feira de Santana \\ tatifarmauefs@yahoo.com.br
}

\begin{abstract}
Resumo
Esse estudo objetivou caracterizar o conhecimento dos Agentes Comunitários de Saúde (ACS) sobre plantas medicinais, por meio de uma pesquisa quantitativa-descritiva realizada em seis Unidades de Saúde da Família, localizadas em um município baiano, no período de março a abril de 2016. Utilizou-se como instrumento de coleta de dados um questionário que foi respondido por 41 do total de 53 ACS. A pesquisa foi aprovada por Comitê de Ética em Pesquisa. O conhecimento sobre plantas medicinais dos participantes foi considerado satisfatório, pois das seis questões apresentadas quatro tiveram acima de 70\% de acertos. Este conhecimento é fundamentado no saber popular, demonstrando a necessidade de educação permanente em saúde. A realização dessa pesquisa foi articulada a atividades de extensão universitária, visando minimizar as lacunas de conhecimentos que foram evidenciadas na pesquisa.

Palavras-chaves: Plantas Medicinais. Estratégia Saúde da Família. Agentes Comunitários de Saúde. Atenção

Primária à Saúde.

\section{KNOWLEDGE OF COMMUNITY HEALTH AGENTS FROM BAHIA-BRAZIL ABOUT MEDICINAL PLANTS}

\begin{abstract}
The aims was to characterize the knowledge of Community Health Agents (ACS) on medicinal plants, through a quantitative-descriptive research carried out in six Family Health Units, located in a neighborhood of a municipality in Bahia. A questionnaire was used as a data collection instrument and was answered by 41 of the 53 ACS. This study was approved by the Research Ethics Committee. For the majority of the questions elaborated on medicinal plants the participants had knowledge considered satisfactory, because of the six questions presented four had above $70 \%$ of correct answers. Despite this, it was verified that the knowledge of community agents about medicinal plants is based on popular knowledge, demonstrating the need for permanent health education to qualify them on the subject. The realization of this study was articulated to university extension activities in order to minimize the knowledge gaps that were highlighted in the research.

Keywords: Medicinal Plants. Family Health Strategy. Community Health Workers. Primary Health Care.

\section{CONOCIMIENTO DE LOS AGENTES COMUNITARIOS DE SALUD EN UN CONDADO DE BAHIA- BRASIL ACERCA DE PLANTAS MEDICINALES}

\footnotetext{
Resumen

Este estudio objetivó caracterizar el conocimiento de los Agentes Comunitarios de Salud-ACS acerca de plantas medicinales, por medio de una investigación cuantitativa-descriptiva realizada en seis Unidades de Salud de la Familia, ubicadas en un barrio de un municipio baiano. Se utilizó como instrumento de recolección de datos un cuestionario que fue respondido por 41 del total de 53 ACS. La investigación fue aprobada por el Comité de Ética en Investigación. Para la mayoría de las cuestiones elaboradas sobre plantas medicinales los participantes tuvieron conocimiento considerado satisfactorio, pues de las seis preguntas presentadas cuatro tuvieron por encima del $70 \%$ de aciertos. Se constató que el conocimiento de los ACS sobre plantas medicinales está fundamentado en el saber popular, demostrando la necesidad de educación permanente en salud. Esta investigación fue articulada a actividades de extensión universitaria, con el objetivo de minimizar las lagunas de conocimientos que fueron evidenciadas en la investigación.

Palabras claves: Plantas Medicinales. Estrategia de Salud de Familiar. Agentes Comunitarios de Salud. Atención Primaria de Salud.
} 
Conhecimento dos agentes comunitários de saúde de um município baiano sobre plantas medicinais

\section{INTRODUÇÃO}

O uso de plantas medicinais como forma de terapia para o tratamento de diversas patologias é comum em várias culturas em todo o mundo. Sua utilização deve-se especialmente ao fato de que a medicina tradicional é baseada em crenças existentes há centenas de anos, antes mesmo do desenvolvimento da medicina moderna e se perpetua a partir da transmissão dos conhecimentos sobre o assunto através das gerações (VEIGA JUNIOR, 2008).

A população brasileira utiliza produtos à base de plantas medicinais nos seus cuidados com a saúde como forma de tratamento profilático, curativo ou paliativo, a partir do conhecimento tradicional (BRASIL, 2012a).

Por definição pode-se chamar de planta medicinal o todo ou a parte de uma espécie vegetal, cultivada ou silvestre, utilizada com finalidade terapêutica. A partir dessas espécies, pode-se obter o medicamento fitoterápico, que pode ser usado na forma simples, quando o ativo é proveniente de uma única espécie vegetal medicinal, ou de forma composta, para substâncias não isoladas (ANVISA, 2014), uma vez que as substâncias que constituem as espécies vegetais podem agir de forma sinérgica ou complementar, favorecendo a efetividade da planta medicinal em sua função (ALMEIDA, 2011).

A Organização Mundial da Saúde tem recomendado a todos os países, principalmente os países em desenvolvimento, ampliar os cuidados de saúde por meio da aplicação das práticas de Medicina Tradicional/Medicina Complementar. A partir dessas recomendações e também de experiências exitosas em regiões brasileiras, bem como a um movimento da sociedade em prol do uso de plantas medicinais, foi instituída em 2006, no Brasil, a Política Nacional de Práticas Integrativas e Complementares (PNPIC), que incluiu o uso de plantas medicinais e outras terapias na atenção básica (BRASIL, 2006a).

Para garantir a qualidade e eficiência dessa prática e com o objetivo de promover o uso adequado e seguro de plantas medicinais pela população brasileira, também foi instituída a Política Nacional de Plantas Medicinais e Fitoterápicos (PNPMF). Essa contém diretrizes que garantem desde o cultivo até a disponibilização dessa forma terapêutica à população, além de promover a preservação da medicina tradicional, uma vez que o uso faz parte dos cuidados primários em saúde (BRASIL, 2006b).

A despeito do importante movimento para implementação dessas políticas, estudo realizado por Badke e colaboradores (2019) revelou que dos 5.570 municípios brasileiros, apenas 126 apresentam algum serviço que utilizavam a fitoterapia como prática integrativa 
Conhecimento dos agentes comunitários de saúde de um município baiano sobre plantas medicinais

e complementar, com registro no Cadastrado Nacional de Estabelecimentos de Saúde (CNES), havendo uma concentração na região Sudeste. Na Bahia, os autores constataram apenas um município (Riachão do Jacuípe) no CNES.

No âmbito da disponibilização desses serviços no Sistema Único de Saúde (SUS), a atenção básica é o espaço mais adequado para implantação das diretrizes e estratégias dessas políticas, pois a equipe de saúde está mais próxima da comunidade, o que possibilita a troca de informações e experiências no que tange aos cuidados com o emprego de plantas medicinais (BRASIL, 2012a).

Muitas patologias comuns na atenção básica respondem bem à fitoterapia como opção terapêutica. De modo geral, a população atendida em unidades básicas de saúde faz uso de plantas medicinais, não conhecendo a possível existência de toxicidade, sua comprovada ação terapêutica, forma correta de cultivo, preparo, indicações e contra indicações. No senso comum a população que utiliza plantas medicinais acredita que por serem de origem vegetal não serão prejudiciais à saúde, independente da forma e quantidade usada (FONTENELE et al., 2013).

Nesse sentido, são de fundamental importância as intervenções dos profissionais que atuam na atenção básica em relação aos cuidados com as plantas medicinais, no intuito de orientar a comunidade sobre seus benefícios e riscos. Em relação aos profissionais das equipes de saúde da família, destaca-se a importância da atuação dos Agentes Comunitários de Saúde (ACS) nas ações de educação em saúde relacionadas às plantas medicinais. A proximidade com as famílias permite o conhecimento do uso dessas terapias, pois muitos usuários não relatam essa utilização para outros profissionais de saúde.

Os ACS são os profissionais identificados pela comunidade como mais relacionados à fitoterapia, pois são pessoas do lugar, que possuem a mesma linguagem da comunidade e carregam certa credibilidade junto à mesma, auxiliando na indicação das plantas medicinais (LEITE; SCHOR, 2005; SCHIAVO; SCHWAMBACH; COLET, 2017).

Os trabalhadores de saúde da atenção básica são os principais responsáveis pelas práticas de educação em saúde voltadas para a comunidade. Desse modo, faz-se necessário que os ACS possuam conhecimentos fundamentais sobre diversas temáticas relacionadas aos cuidados em saúde, dentre eles o uso de plantas medicinais. Tais conhecimentos permitem que esses trabalhadores tenham uma melhor atuação junto aos usuários orientando-os sobre benefícios das plantas medicinais, modos de preparo, toxicidade e o seu uso racional, garantindo que essa terapia seja segura, preservada, resolutiva e eficiente. Assim, este estudo teve como objetivo caracterizar o conhecimento dos ACS sobre plantas 
Conhecimento dos agentes comunitários de saúde de um município baiano sobre plantas medicinais

medicinais de modo a identificar as possíveis lacunas desse conhecimento para subsidiar ações de educação permanente direcionadas a esses trabalhadores.

\section{METODOLOGIA}

Trata-se de uma pesquisa quantitativa-descritiva, realizada em seis Unidades de Saúde da Família (USF) localizadas em um bairro de um município baiano, no período de março a abril de 2016. As USF foram escolhidas intencionalmente pelos pesquisadores por serem campos de práticas de várias componentes curriculares da Universidade Estadual de Feira de Santana e estarem incluídas no campo de atuação do Programa de extensão universitário intitulado Programa de promoção do uso adequado de plantas medicinais e fitoterápicos pela população dos municípios do semiárido baiano, na perspectiva de articular a pesquisa, o ensino e a extensão.

Os participantes da pesquisa foram 41 ACS que trabalham nessas unidades. De acordo com o Cadastro Nacional de Estabelecimentos de Saúde - CNES existia, no momento da coleta de dados, 53 ACS, para as seis unidades. No entanto, 12 ACS não participaram da pesquisa, pois 10 estavam afastados do trabalho por motivo de licença, e dois não aceitaram participar.

Os dados foram produzidos por meio de um questionário, elaborado pelos próprios pesquisadores, com perguntas fechadas e abertas sobre: os conceitos, utilização, preparo e indicação de plantas medicinais, fonte de informações utilizadas e formas de capacitação sobre o tema na unidade. Estes foram respondidos após consentimento dos participantes e da assinatura do Termo de Consentimento Livre e Esclarecido (TCLE).

Os dados foram organizados em um banco computadorizado e processados através do software Statiscal Package for Social Sciences - SPSS, versão 20.0. A análise de dados foi realizada utilizando a estatística descritiva, que geraram gráficos e tabelas, e tendo como base referencial teóricos da área de estudo sobre plantas medicinais.

A pesquisa obedeceu a Resolução 466, de 12 de dezembro de 2012 (BRASIL, 2012b) e foi aprovada pelo Comitê de Ética em Pesquisa (CEP) da Universidade Estadual de Feira de Santana (UEFS), autorizada pelo Parecer CEP/UEFS n ${ }^{\circ} .440 .717$. 
Conhecimento dos agentes comunitários de saúde de um município baiano sobre plantas medicinais

\section{RESULTADOS E DISCUSSÃO}

As questões relacionadas à temática plantas medicinais buscaram caracterizar o conhecimento dos ACS em relação a importantes informações que os mesmos devem possuir para atuarem junto à comunidade, de modo a promover ações de educação em saúde com embasamento científico sobre o uso dessa terapia. Foram realizados alguns questionamentos com resposta do tipo verdadeiro ou falso. Cada questão foi analisada de acordo com a literatura científica, estão descritas abaixo, e sintetizadas na Tabela 1.

TABELA 1: Conhecimentos dos ACS sobre plantas medicinais

\begin{tabular}{|c|c|c|c|c|c|}
\hline Questões & $\begin{array}{l}\text { Respostas de } \\
\text { acordo com a } \\
\text { literatura }\end{array}$ & $\begin{array}{l}\text { Referência da } \\
\text { literatura }\end{array}$ & $\begin{array}{c}\text { Acertos } \\
(\%)\end{array}$ & $\begin{array}{l}\text { Erros } \\
(\%)\end{array}$ & $\begin{array}{l}\text { Percentual de } \\
\text { questões não } \\
\text { respondidas }\end{array}$ \\
\hline $\begin{array}{l}\text { Você acha que as plantas } \\
\text { medicinais podem ajudar na saúde } \\
\text { do paciente? }\end{array}$ & Verdadeiro & $\begin{array}{l}\text { SCHULZ; HANSEL; } \\
\text { TYLER, } 2002 .\end{array}$ & 97,6 & 2,4 & 0,0 \\
\hline $\begin{array}{c}\text { Você acha que as plantas } \\
\text { medicinais, se não fizer bem, mal } \\
\text { não faz? }\end{array}$ & Falso & $\begin{array}{l}\text { VEIGA JUNIOR; } \\
\text { PINTO, MACIEL, } \\
2005\end{array}$ & 70,7 & 26,8 & 2,4 \\
\hline $\begin{array}{c}\text { Droga vegetal, planta medicinal e } \\
\text { fitoterápico são nomes como o } \\
\text { mesmo significado? }\end{array}$ & Falso & ANVISA, 2014 & 56,1 & 39,0 & 4,9 \\
\hline $\begin{array}{l}\text { Você acha que a única maneira de } \\
\text { preparar um chá é cozinhar as } \\
\text { folhas por alguns minutos? }\end{array}$ & Falso & OSUNA et al., 2005 & 95,1 & 4,9 & 0,0 \\
\hline $\begin{array}{l}\text { Você acha que durante a gravidez é } \\
\text { mais indicado consumir chás para } \\
\text { tratar mal estar da gravidez, pois } \\
\text { eles representam um menor risco à } \\
\text { saúde? }\end{array}$ & Falso & $\begin{array}{l}\text { CLARKE; RATES; } \\
\text { BRIDI, } 2007\end{array}$ & 85,4 & 9,8 & 4,9 \\
\hline $\begin{array}{l}\text { Não há problemas em utilizar } \\
\text { plantas medicinais juntamente com } \\
\text { medicamentos? }\end{array}$ & Falso & $\begin{array}{l}\text { NICOLETTI et al., } \\
\text { 2007; NICOLETTI; } \\
\text { PINTO, } 2010\end{array}$ & 41,5 & 56,1 & 2,4 \\
\hline
\end{tabular}

Fonte: Os autores

Em seguida serão apresentadas as discussões relacionadas a cada uma das questões que foram julgadas pelo ACS.

\section{AS PLANTAS MEDICINAIS PODEM AJUDAR NA SAÚDE DO PACIENTE?}

A confiança na terapia com plantas medicinais foi demonstrada pelos ACS, sendo que $97,6 \%$ desses acreditam nos seus benefícios. Apesar dos avanços no saber na área de saúde e do uso de medicamentos convencionais, a população acredita que as plantas medicinais são uma terapia eficaz. Isso se deve em parte ao fato do uso dessa terapia no 
Conhecimento dos agentes comunitários de saúde de um município baiano sobre plantas medicinais

Brasil ser um hábito estabelecido culturalmente, devido às diversas origens étnicas dos colonizadores desse país (ALMEIDA, 2011) e também de seu próprio povo.

O valor medicinal do uso de plantas, utilizadas em forma de chás, é largamente baseado no saber popular. No entanto, há estudos científicos para diversas drogas vegetais, em forma de extratos com comprovação dos seus efeitos benéficos para a saúde. Têm-se como exemplos, drogas vegetais como extrato de Ginko Biloba eficaz no tratamento sintomático de deficiências cognitivas; o extrato da raiz de Piper methysticum (Kawa kawa) eficaz como droga ansiolítica; a castanha da índia e várias plantas que são utilizadas para distúrbios do aparelho circulatório; Eucalyptus s.p (eucalipto) e Salix alba (salgueiro) para problemas no aparelho respiratório; e a ação laxativa das plantas com antranóides para distúrbios no aparelho digestivo; e a Mentha spicata (hortelã) para má digestão entre outras (SCHULZ; HANSEL; TYLER, 2002).

O motivo da utilização da Medicina Tradicional Complementar (que contempla o uso de plantas medicinais) está relacionado a alguns fatores como: ser a principal fonte de atenção à saúde e muitas vezes a única; estar perto das casas, sendo acessível, culturalmente aceito, confiável e, portanto, mais uma alternativa para lidar com o aumento incessante das doenças crônicas não transmissíveis (WHO, 2013).

Rates (2001) aponta outros motivos do interesse da população pelas plantas medicinais como os resultados negativos que muitas vezes se obtém com os medicamentos convencionais, seus efeitos adversos e a impossibilidade de cura, a dificuldade no acesso aos medicamentos e a medicina institucionalizada, a consciência ecológica e a crença de que o natural é inofensivo.

Ao analisar o histórico do uso das plantas medicinais, evidencia-se que há confiança da população na eficácia das plantas medicinais devido à adesão familiar com este tratamento. Consequentemente, a simbologia e a ideologia do paciente sobre a segurança do uso de plantas medicinais são um forte instrumento para sua incorporação à terapêutica (SCHULZ; HANSEL; TYLER, 2002), constituindo-se, portanto, uma vantagem dessa terapia diante do uso de fármacos de origem sintética (ALMEIDA, 2011). Esta compreensão é também ratificada em outros estudos sobre utilização de plantas medicinais (BADKE et al., 2016; SCHIAVO; SCHWAMBACH; COLET, 2017)

Estudo realizado com moradores da comunidade da Barra do Jucu, no município de Vila Velha-ES demonstrou que as pessoas que tratavam seus problemas de saúde com as plantas consideravam um tratamento mais lento, porém essas acreditavam que essa terapia causa menos mal ou não faz mal, alguns usam para manter a tradição ou pela 
Conhecimento dos agentes comunitários de saúde de um município baiano sobre plantas medicinais

comodidade de ter as plantas no quintal, outros por ser uma alternativa acessível e alguns acreditam que as plantas têm mais poder e eficiência que os medicamentos convencionais (ALBERTASSE et al., 2010).

A confiança da população brasileira nas plantas medicinais evidencia a necessidade de prover políticas públicas que regulem, promovam o uso adequado de plantas medicinais e preservem esse conhecimento popular (BRASIL, 2012a). Nesta perspectiva, ressalta-se que muitos estados e municípios estabeleceram políticas específicas para uso adequado de plantas medicinais.

\section{O USO DAS PLANTAS MEDICINAIS, SE NÃO FIZER BEM, MAL NÃO FAZ?}

A resposta dos ACS a essa questão demonstrou que 70,7\% reconhecem que há riscos no uso de plantas medicinais. Apesar dos seus benefícios e da confiança da população nessa terapia, a sua utilização não é isenta de riscos à saúde, porém no senso comum as pessoas acreditam no mito "se é natural não faz mal". No entanto, essas podem causar diversas reações como intoxicações, enjoos, irritações, edemas e até a morte, como qualquer outro medicamento (BRASIL, 2010).

Veiga Junior, Pinto e Maciel (2005) destacam algumas plantas medicinais que podem causar efeitos adversos tais como: Alho (Allium sativum) - náuseas, vômitos; Aloe (Aloe ferox) - desconforto abdominal; Angélica (Angelica archangelica) - fotodermatite; Anis (Pimpinella anisum) - dermatite por contato; Boldo (Peumus boldo) - irritação renal; Capsicum (Capsicum annum) - alveolite alérgica; Cássia (Cinnamomum cássia) - reações alérgicas; Confrei (Symphytum officinale) - hepatotoxicidade; Dente-de-leão (Taraxacum officinale) reações alérgicas por contato; Erva-de-São-João (Hypericum perforatum) - fotodermatite; Mate (Ilex paraguaiensis) - distúrbios hepáticos; Sene (Cassia angustifólia) - desconforto abdominal.

Estudo realizado com um grupo de pacientes diabéticos inseridos no Programa de Hiperdia em um Centro de Saúde no município de Jequié-BA, detectou que 66\% dos entrevistados usam as plantas medicinais por achar que não fazem mal à saúde (SILVA et al., 2008). Pesquisa com usuários de Unidades Básicas de Saúde do município de Fátima do Sul-MS, revelou que ainda há pessoas que acreditam que as plantas medicinais são incapazes de provocar malefícios (BIN et al., 2007).

A crença popular de que plantas medicinais não oferecem riscos se deve a diversos fatores tais como a informalidade como essas plantas medicinais e os seus derivados são comercializados, falhas de fiscalização e regulamentação por parte da vigilância sanitária, 
Conhecimento dos agentes comunitários de saúde de um município baiano sobre plantas medicinais

falta de conhecimento dos riscos potenciais envolvidos no consumo dessas plantas por parte daqueles que as usam indiscriminadamente e as propagandas de massa que exploram a ideia do que é natural não faz mal (SILVA et al., 2008).

Os estudos citados acima tiveram como participantes a população em geral, leigos desprovidos de tais conhecimentos e que não trabalham na área da saúde. Neste estudo, em particular, esperava-se um percentual mais alto de acerto em relação a essa questão, já que se dirigiu aos ACS que são profissionais de saúde. Em se tratando desses trabalhadores, alguns mitos e erros propagados no senso comum já deveriam ter sido desconstruídos pelo fato desses fazerem parte da equipe de saúde da família e ter sua atuação voltada às práticas de promoção e prevenção da saúde, onde o encontro do saber popular e do saber científico deve ser problematizado de modo a trazer benefícios à comunidade.

\section{DROGA VEGETAL, PLANTA MEDICINAL E FITOTERÁPICO SÃO NOMES COM O MESMO SIGNIFICADO?}

Em relação aos conceitos de plantas medicinais, 56,1 \% dos ACS afirmaram que há diferença entre os termos droga vegetal, planta medicinal e fitoterápico e 39\% desses desconhecem essas diferenças. O conhecimento de tais conceitos podem demostrar a compreensão das aplicabilidades dessas terapias. Esses termos são expostos na Resolução da Diretoria Colegiada (RDC) da Anvisa no 26, de 13 de maio de 2014, que dispõe sobre o registro de medicamentos fitoterápicos e o registro e a notificação de produtos tradicionais fitoterápicos (ANVISA, 2014):

\footnotetext{
VIII - Droga vegetal: planta medicinal, ou suas partes, que contenham as substâncias responsáveis pela ação terapêutica, após processos de coleta/colheita, estabilização, quando aplicável, e secagem, podendo estar na forma íntegra, rasurada, triturada ou pulverizada. [...]

XI - Fitoterápico: produto obtido de matéria prima ativa vegetal, exceto substâncias isoladas, com finalidade profilática, curativa ou paliativa, incluindo medicamento fitoterápico e produto tradicional fitoterápico, podendo ser simples, quando o ativo é proveniente de uma única espécie vegetal medicinal, ou composto, quando o ativo é proveniente de mais de uma espécie vegetal. [...]

XXIII - Planta medicinal: espécie vegetal, cultivada ou não, utilizada com propósitos terapêuticos.
}

Existem três formas principais de se usar a Fitoterapia: 1) uso de medicamentos fitoterápicos industrializados; 2) uso de medicamentos fitoterápicos manipulados; 3) uso das plantas medicinais, mediante preparações caseiras (FIGUEREDO; GURGEL; GURGEL JÚNIOR, 2014). Assim, demonstra-se que por não compreender a diferença, os 
Conhecimento dos agentes comunitários de saúde de um município baiano sobre plantas medicinais

ACS desconhecem os conceitos básicos sobre o uso de plantas medicinais, indicando terem como embasamento teórico apenas o senso comum.

\section{A ÚNICA MANEIRA DE PREPARAR UM CHÁ É COZINHAR AS FOLHAS POR} ALGUNS MINUTOS?

O estudo demonstrou que $95,1 \%$ do ACS sabem que o preparo das plantas medicinais não é apenas pelo método de decocção, que consiste em colocar a planta na água fria até a fervura. Essa é uma das maneiras pelos quais os chás podem ser preparados; outras formas são a infusão, que consiste em verter água fervente sobre a droga vegetal e, em seguida, tampar ou abafar o recipiente por um período de tempo determinado; e a maceração, método mais indicado para drogas vegetais que possuam substâncias que se degradem com o aquecimento (ANVISA, 2014).

A decocção é modo mais indicado para preparar chás a partir de talos e raízes, uma vez que, se utilizado no preparo de chás com folhas, flores e sementes, o calor prolongado pode resultar na desestruturação dos metabólitos secundários responsáveis pelos efeitos terapêuticos das plantas medicinais (OSUNA et al., 2005). O chá por decocção é a forma mais citada nos estudos etnobotânicos brasileiros publicados entre os anos de 2009 e 2014 (SANTOS et al., 2017).

Em um estudo realizado por Giraldi e Hanazaki (2010) com moradores do Sertão do Ribeirão em Florianópolis-SC, constatou-se que a indicação da parte da planta utilizada para fins medicinais mais referida foi a folha $(68 \%)$, geralmente para a preparação de chá, por decocção $(66 \%)$. De forma semelhante, pesquisa com uma amostra da população de Datas-MG apontou que 376 entrevistados $(75,2 \%)$ preparam as plantas medicinais em forma de chá (decocção) (ARNOUS et al., 2005). Em ambos os estudos, a maioria dos entrevistados não conhece a maneira correta de preparo das plantas medicinais, devido ao fato de que, na maioria das vezes, a planta é utilizada de forma incorreta, pois somente as partes duras (raiz, caule e casca) devem ser cozidas. Desse modo, é importante o conhecimento das diversas formas de preparo da planta medicinal para que a sua eficácia seja preservada e se tenha segurança na utilização. 
Conhecimento dos agentes comunitários de saúde de um município baiano sobre plantas medicinais

DURANTE A GRAVIDEZ É MAIS INDICADO CONSUMIR CHÁS PARA TRATAR MAL ESTAR DA GRAVIDEZ, POIS ELES REPRESENTAM UM MENOR RISCO À SAÚDE?

Observou-se que 85,4\% dos ACS responderam que é falsa a informação de que na gravidez é mais indicado consumir chás, pois eles representam um menor risco à saúde. Clarke et al., (2007) apontam que, para a maioria das plantas medicinais, não há dados a respeito da segurança de uso durante a gravidez, sendo os dados existentes escassos e muitas vezes contraditórios. Informação também mencionada na maioria das monografias presentes no Memento Fitoterápico da Farmacopeia Brasileira (ANVISA, 2016), corroborando a necessidade de cuidados no uso de plantas medicinais por gestantes.

Apesar da escassez de estudos, há evidências que algumas plantas não devem ser utilizadas durante a gravidez por estimularem a motilidade uterina e poderem provocar aborto tais como: alho (Allium sativum), aloe (Aloe ferox), angélica (Angelica archangelica), arnica (Arnica montana), cânfora (Cinnamomum canphora), confrei (Symphitum officinalis), eucalipto (Eucaliptus globulus), alecrim (Rosmarinus officinalis), gengibre (Zengiber officinalis) e sene (Cassia angustifolia e Cassia acutifolia). Alguns óleos essenciais também devem ser evitados, como exemplo, os provenientes de bétula (Betula alba), cedro (Cedrela brasiliensis), erva-doce (Pimpinella anisum), jasmim (Jasminum officinalis), manjericão (Origanum basilicum), manjerona (Majorana hortensis), tomilho (Thymus vulgaris), rosa (Rosa sp.) e lavanda (Lavanda angustifólia) (VEIGA JUNIOR; PINTO; MACIEL, 2005).

Rangel e Bragança (2009) analisaram as representações formadas sobre o uso de plantas medicinais por gestantes em tratamento ambulatorial em Niterói-RJ, onde as gestantes que utilizavam o faziam com base nas informações de familiares, por serem preparações mais simples. Mesmo aquelas gestantes que não utilizavam plantas medicinais, desconheciam a fitoterapia, ou não compreendiam que o uso de plantas medicinais poderia acarretar riscos para si mesma e para o feto, apresentavam dificuldades de identificação e administração segura das plantas e certo grau de mistificação, além de usarem substâncias ineficazes e potencialmente tóxicas. Os autores alertam para a necessidade de maior diálogo dos trabalhadores de saúde com as gestantes, no sentido de informar às mulheres em idade fértil sobre o risco, assim como sobre possibilidades da utilização de plantas como medicamentos na gravidez.

A principal orientação para as mulheres grávidas é não utilizar qualquer medicamento (VEIGA JUNIOR; PINTO; MACIEL, 2005; CLARKE; RATES; BRIDI, 2007), seja ele de origem vegetal ou sintética, sem o conhecimento prévio de um 
Conhecimento dos agentes comunitários de saúde de um município baiano sobre plantas medicinais

profissional. Portanto, cabe aos profissionais da saúde orientar as mulheres sobre o risco da utilização de planta medicinal e fitoterápico na gravidez, ressaltando para o perigo potencial da automedicação irresponsável.

Nesse sentido, faz-se necessário que os ACS conheçam as gestantes do seu território e as alertem sobre os riscos da utilização de plantas na gravidez.

\section{NÃO HÁ PROBLEMAS EM UTILIZAR PLANTAS MEDICINAIS JUNTAMENTE COM MEDICAMENTOS?}

Constatou-se que 56,1\% dos ACS afirmaram não haver problemas em utilizar plantas medicinais juntamente com medicamentos. De acordo com as recomendações do Ministério da Saúde e órgãos reguladores não é aconselhado associações de medicamentos com plantas devido ao risco de interação de plantas medicinais com medicamentos (BRASIL, 2010), pois a depender da planta medicinal que se utiliza não há estudos científicos sobre interações dessas com os medicamentos. Em caso de interações já relatadas na literatura científica é necessário que o profissional de saúde as conheça e oriente o usuário sobre a possibilidade ou não do uso das plantas medicinais juntamente com os medicamentos.

Porém, é necessário que os profissionais de saúde estimulem os usuários a relatarem sobre as plantas medicinais que estão utilizando, pois é comum a falta de comunicação entre prescritor e o usuário sobre o uso dessa terapia. O nível de desconhecimento do médico só aumenta os riscos do paciente, uma vez que pode haver imprecisão no diagnóstico em função das muitas interações possíveis entre as plantas e os medicamentos da medicina convencional (VEIGA JUNIOR; PINTO; MACIEL, 2005).

Um estudo de revisão de literatura científica apresentou as principais interações de 48 drogas de origem vegetal que são muito utilizadas e apresentam interesse na cultura popular, sendo que grande parte dessas fazem parte da Lista de Medicamentos Fitoterápicos de Registro Simplificado de 2008 (NICOLET'TI et al., 2010).

Tem-se como exemplos de interações medicamentosas com plantas medicinais: alho (Allium sativum L.) com drogas hipoglicemiantes como a insulina e a glipizida, causando hipoglicemia; o boldo e boldo do Chile (Peumus boldo Molina) interagem com medicamentos anticoagulantes, aumentando a função antiplaquetária; a camomila (Matricaria recutita L.) interage com anticoagulantes, como a varfarina, aumentando o risco de sangramento, com barbitúricos (fenobarbital) podendo intensificar ou prolongar a ação depressora do sistema nervoso central e com o ferro, reduzindo sua absorção; a cáscara 
Conhecimento dos agentes comunitários de saúde de um município baiano sobre plantas medicinais

sagrada (Aesculus hippocastanum L.) aumenta o risco de sangramentos quando utilizada com ácido acetilsalicílico, varfarina, heparina, clopidogrel e anti-inflamatórios como ibuprofeno ou naproxeno; a Erva de São João (Hypericum perforatum L.) interage com o omeprazol, talbutamida, cafeína, carbamazepina, ciclosporina, midazolam, nifedipina, sinvastatina, teofilina, antidepressivos tricíclicos, varfarina, inibidores da transcriptase reversa não nucleosídeos, inibidores da protease e contraceptivos orais (NICOLETTI et al., 2010).

O uso das plantas medicinais pode causar interações com os medicamentos que são metabolizados pelo sistema hepático enzimático, intensificando o efeito ou reduzindo-o, pode também afetar a absorção de medicamentos e por esses motivos deve-se conhecer esses possíveis riscos para promover o uso adequado de plantas medicinais (NICOLETTI et al., 2007).

Para a maioria das questões fundamentais sobre plantas medicinais os ACS tiveram um conhecimento satisfatório, pois das seis questões apresentadas quatro tiveram acima de $70 \%$ de acerto, o que mostra uma consciência sobre o uso adequado de plantas medicinais. No entanto, algumas informações necessárias para a garantia do uso racional de plantas medicinais, devem ser de conhecimento de todo ACS, haja vista seu importante trabalho na equipe de saúde da família, com atuação principalmente na promoção e prevenção da saúde.

\section{FONTES DE INFORMAÇÃO SOBRE PLANTAS MEDICINAIS E EDUCAÇÃO PERMANENTE EM SAÚDE}

Além das questões relacionadas aos conhecimentos fundamentais de plantas medicinais foram questionados aos ACS sobre fontes de informações sobre plantas medicinais e conhecimento sobre as políticas de saúde relacionadas a plantas medicinais e Fitoterapia e a participação em atividades de educação em saúde.

As fontes de informação sobre plantas medicinais mais referidas foram familiares, amigos, curandeiros e TV, somando 69,4\% (Tabela 2). Assim, entende-se que os ACS obtêm informações de fontes sem comprovação científica. 
Conhecimento dos agentes comunitários de saúde de um município baiano sobre plantas medicinais

TABELA 2 - Fonte de informações dos ACS sobre plantas medicinais

\begin{tabular}{lr}
\hline \hline Fonte de informações sobre plantas medicinais & Percentual \\
\hline Familiares & 33,7 \\
Amigos & 19,8 \\
Curandeiros & 1,0 \\
Médicos & 1,0 \\
Livros especializados & 22,8 \\
Outros profissionais de saúde & 6,9 \\
TV & 14,9 \\
Total & 100,0 \\
\hline \hline Fonte: Os autores
\end{tabular}

De modo similar, o estudo de Martins, Pereira e Ceolin (2008) mostrou que o conhecimento dos ACS sobre plantas medicinais é baseado no conhecimento tradicional, demostrando a necessidade de educação permanente, de modo a melhorar os serviços de saúde relacionados às plantas medicinais, incluindo a utilização de fontes com comprovação científica.

Um percentual significativo de ACS (90,3\%), não conhecem os programas e/ou políticas relacionadas a plantas medicinais, sendo as mais recentes: a Política Nacional de Práticas Integrativas e Complementares (PNPIC); a Política Nacional de Plantas Medicinais e Fitoterápicos; o Programa Nacional de Plantas Medicinais e Fitoterápicos; e o Programa Farmácia Viva no âmbito do Sistema Único de Saúde (BRASIL 2006a; BRASIL 2006b; BRASIL, 2010).

O desconhecimento dessas políticas e programas pelos gestores e trabalhadores de saúde também foi demonstrado por estudo de Araújo et al., (2014), no qual 81\% dos entrevistados, diretores de Unidades de Saúde da Família, desconheciam as normativas vigentes relacionadas à Fitoterapia no âmbito das políticas nacionais de saúde.

Em uma pesquisa realizada com os ACS das equipes de saúde da família de USF da zona urbana e rural de Feira de Santana-BA, observou que as políticas públicas e programas relacionados ao tema também são desconhecidos (BRITO; SANTOS; ALENCAR, 2015). Outro estudo realizado em dois municípios do semiárido baiano mostrou que o conhecimento sobre as Práticas Integrativas e Complementares (PIC) foi superficial entre os gestores (FILHO; OLIVEIRA; NASCIMENTO, 2015).

A falta de divulgação dessas políticas e programas resulta no desconhecimento por parte dos profissionais de saúde e dos gestores e, consequentemente, não são realizadas ações em prol da implantação dessas práticas na atenção básica ou quando ocorre é de forma incipiente (DUTRA, 2009; MATSUCHITA; MATSUCHITA, 2015). 
Conhecimento dos agentes comunitários de saúde de um município baiano sobre plantas medicinais

Em relação à participação dos ACS em atividades de educação permanente com a temática de plantas medicinais, 51\% afirmaram ter participado de alguma atividade. Analisando esse dado e com base nas ações já realizadas nas unidades pesquisadas, entende-se que não há um programa de educação permanente que contemple temas referentes às ações, serviços, programas e políticas relacionadas às plantas medicinais.

A educação permanente, prevista na Política Nacional de Atenção Básica, requer compartilhamento de saberes, práticas intersetoriais e de gestão do cuidado em rede. Estas atividades precisam de um planejamento educativo ascendente com identificação de nós críticos de modo coletivo a serem atrelados, para que esses possam ser problematizados em espaço pedagógico (BRASIL, 2017). Desse modo, percebe-se uma ampliação de discussões sobre o uso de plantas medicinais no âmbito nacional, entretanto, no nível local pesquisado, essa temática não tem sido considerada uma prioridade.

Em geral, apesar da forma heterogênea como esse tema tem sido debatido e desenvolvido no país, conforme tem sido identificado pelos estudos, há uma perspectiva positiva em relação à efetivação de políticas de saúde que promovam o uso racional de plantas medicinais e de outras terapias. Isso porque muitos municípios e estados têm se debruçado para a elaboração e instituição de políticas adequadas às realidades local e regional, institucionalizando práticas que já vinham sendo produzidas nos territórios.

\section{CONSIDERAÇÕES FINAIS}

O ACS é um profissional diferenciado na equipe de saúde da família por apresentar a função de trabalhador de saúde e membro da comunidade. Essa peculiaridade lhe permite ser o interlocutor entre conhecimento científico que se encontra no sistema formal dos serviços de saúde e o saber popular que tem o seu devido valor, por advir das experiências cotidianas de saúde/doença dos usuários. Nesse sentido, o conhecimento sobre saúde/doença dos ACS é fruto dessa articulação que é muito importante, porém deve ser problematizado para que ambos se complementem e promovam saúde para a comunidade atendida.

Este estudo permitiu concluir que os ACS das seis unidades apresentam algum conhecimento sobre o uso racional de plantas medicinais, visto que quando comparados com outras pesquisas realizadas com a população em geral, demonstraram maior compreensão sobre o tema. No entanto, identificou-se que não há uma prática de educação permanente em saúde dirigida aos ACS em relação à temática. Estes possuem meios não 
Conhecimento dos agentes comunitários de saúde de um município baiano sobre plantas medicinais

científicos como fontes de informações e a maioria desconhecem as políticas e programas relacionados a plantas medicinais.

Entre as possíveis limitações do estudo estão os questionamentos elegidos para compor o instrumento de coleta de dados, já que outras perguntas também importantes podem não ter constado. Tal ponderação foi feita, pois o instrumento precisava ter um quantitativo razoável de perguntas para garantir melhor adesão dos respondentes. Outro aspecto foi a seleção intencional das unidades pesquisadas, que se justificou pela necessidade de garantir a viabilidade do estudo.

Portanto, pode-se caracterizar o conhecimento dos ACS fundamentado no saber popular, necessitando de melhor embasamento científico para atuarem de modo mais adequado nas atividades de educação em saúde da população. Esse estudo apontou para a necessidade de educação permanente sobre plantas medicinais, principalmente quanto a conceitos gerais, plantas tóxicas, interações entre plantas medicinais e medicamentos, uso de plantas em grupos específicos como gestantes, idosos e crianças e fonte de informação sobre plantas medicinais.

A devolutiva dos resultados dessa pesquisa consistiu na execução de um plano de trabalho do Programa de promoção do uso adequado de plantas medicinais e fitoterápicos pela população dos municípios do semiárido baiano - programa de extensão da Universidade Estadual de Feira de Santana (UEFS), para desse modo atuar de forma contínua na promoção da educação em saúde sobre o tema plantas medicinais para trabalhadores das equipes de saúde da família e para os usuários.

Com a intenção de promover o uso adequado de plantas medicinais, o programa de extensão realizou seis capacitações de 3 horas de duração cada uma, voltada para os ACS de cada USF. Durante as capacitações intituladas "Plantas medicinais: informações essenciais para os $A C S$ ” se discutiu sobre a importância das plantas medicinais, políticas públicas, plantas para os distúrbios menores, cuidados com o uso de plantas medicinais e plantas tóxicas. Utilizou-se para isso um álbum seriado, elaborado para uso nas unidades de saúde e também foram distribuídos materiais educativos impressos: um folder intitulado "Formas de uso das plantas medicinais" e uma cartilha denominada "Plantas medicinais" que continham o nome popular e científico, a imagem das plantas, as indicações de uso, a posologia recomendada e as contraindicações de uso de plantas medicinais comuns no semiárido baiano.

Dessa forma, houve um compartilhamento de saberes e uma relação entre pesquisa e extensão em prol do uso racional de plantas medicinais. Ademais, a pesquisa e as 
Conhecimento dos agentes comunitários de saúde de um município baiano sobre plantas medicinais

atividades realizadas motivaram a discussão do tema no município de estudo, antes inexistente ou ainda incipiente em algumas unidades de saúde.

\section{REFERÊNCIAS}

ALBERTASSE P.D, THOMAZ L.D., ANDRADE M.A. Plantas medicinais e seus usos na comunidade da Barra do Jucu, Vila Velha, ES. Rev. Bras. Plantas Med., Paulínea, v. 3, n. 32, p. 250-260, 2010.

ALMEIDA, M.Z. Plantas Medicinais. 3ed. Salvador: EDUFBA, 2011.

ANVISA. Agência Nacional de Vigilância Sanitária. RDC no 26, de 13 de maio de 2014. Dispõe sobre o registrode medicamentos fitoterápicos e o registro e a notificação de produtos tradicionais fitoterápicos. Diário Oficial da da União, Poder Executivo, Brasília, DF, 14 de maio de 2014.

ANVISA. Agência Nacional de Vigilância Sanitária. RESOLUÇÃO DA DIRETORIA COLEGIADA - RDC Nº. 84, DE 17 DE JUNHO DE 2016. Aprova o Memento Fitoterápico da Farmacopeia Brasileira e dá outras providências. Disponível em: <http://portal.anvisa.gov.br/documents/33832/2909630/Memento+Fitoterapico/a80ec4 77-bb36-4ae0-b1d2-e2461217e06b >. Acesso em 07 dez. 2019

ARAÚJO, W. R. M, et al. Inserção da Fitoterapia em unidades de saúde da família de São Luís, Maranhão: realidade, desafios e estratégias. Rev. Bras. Med. Fam. Comunidade, Rio de Janeiro, v. 9, n. 32, p.258-263, 2014.

ARNOUS, A.H; SANTOS, A.S; BEINNER, R.P.C. Plantas medicinais de uso caseiro conhecimento popular e interesse por cultivo comunitário. Rev. Espaç. Saúde, Londrina, v. 6, n. 2, p. 1-6, 2005.

BADKE, M. R. et al. Saber popular: uso de plantas medicinais como forma terapêutica no cuidado à saúde. Rev. Enferm UFSM, v. 6, n.2, p. 225-234, 2016. Disponível em: <https://periodicos.ufsm.br/reufsm/article/view/33655/pdf>. Acesso em: 07 dez. 2019.

BIN, M.C. et al. Conhecimento sobre utilização de plantas medicinais por Pacientes do sistema único de saúde de Fátima do Sul, MS. Interbio, Dourados, v. 1 n. 2, p.1-9, 2007.

BRASIL. Portaria Interministerial no 971, de 3 de maio de 2006. Dispõe sobre a aprovação da política nacional das práticas integrativas e complementares no Sistema Único de Saúde. Diário Oficial da União, Poder Executivo, Brasília, DF, 4 maio 2006a. Seção 1, p. 20.

BRASIL. Decreto n ${ }^{\circ}$ 5.813, de 22 de junho de 2006. Aprova a Política Nacional de Plantas Medicinais e Fitoterápicos e dá outras providências. Diário Oficial da União, Poder executivo, Brasília, DF, 23 jun 2006b, seção 2, p.2. Disponível em:

BRASIL. Ministério da Saúde, Agência Nacional de Vigilância Sanitária. O que devemos saber sobre medicamentos. Brasília-DF: Ministério da Saúde, 2010. 
Conhecimento dos agentes comunitários de saúde de um município baiano sobre plantas medicinais

BRASIL. Portaria n $\mathbf{n}^{\circ}$.436, de 21 de setembro de 2017. Aprova a Política Nacional de Atenção Básica, estabelecendo a revisão de diretrizes para a organização da Atenção Básica, no âmbito do Sistema Único de Saúde (SUS). Brasília-DF: Ministério da Saúde, 2017. Disponível em: http://bvsms.saude.gov.br/bvs/saudelegis/gm/2017/prt2436 2209 2017.html. Acesso em: 06 jun. 2019.

BRASIL. Ministério da Saúde. Secretaria de Atenção à Saúde. Departamento de Atenção Básica. Práticas integrativas e complementares: plantas medicinais e fitoterapia na Atenção Básica. Brasília-DF: Ministério da Saúde, 2012 a. Disponível em: http://bvsms.saude.gov.br/bvs/publicacoes/praticas integrativas complementares planta s medicinais cab31.pdf. Acesso em: 06 jun. 2019.

BRASIL. Resolução no 466, de 12 de dezembro de 2012. Conselho Nacional de Saúde. Aprova as diretrizes e normas regulamentadoras de pesquisa envolvendo seres humanos. Diário Oficial da União, Poder executivo, Brasília, 13jun. 2012b. Seção 1, p.59.

BRITO, D. S.; SANTOS, E. C.; ALENCAR, B. R. Fitoterapia na Estratégia Saúde da Família: percepção dos Agentes Comunitários de Saúde. OLIVEIRA, L. C. F.; NASCIMENTO M. A. A. Práticas integrativas e complementares de saúde no semiárido baiano: utopia ou realidade. Feira de Santana: UEFS Editora; 2017. p.115-144.

CLARKE, J. H. R.; RATES, S. M. K.; BRIDI, R. Um alerta sobre o uso de produtos de origem vegetal na gravidez. Infarma, Brasília, v.19, n.1/2, p.41-8, 2007.

DUTRA, M. G. Plantas medicinais, fitoterápicos e saúde pública: um diagnóstico situacional em Anápolis, 2009. 112 f. Dissertação (Mestrado Multidisciplinar em Sociedade, Tecnologia e Meio Ambiente) Programa de Pós-Graduação em Sociedade, Tecnologia e Meio Ambiente. Centro Universitário de Anápolis, Goiás, 2009.

FIGUEREDO, C. A.; GURGEL, I. G. D.; GURGEL JÚNIOR, G. D. A Política Nacional de Plantas Medicinais e Fitoterápicos: construção, perspectivas e desafios. Physis, Rio de Janeiro, v. 24, n.2, p. 381-400, 2014.

FILHO, A. C. S. A.; OLIVEIRA, L. C. F.; NASCIMENTO M. A. A. Práticas Integrativas e Complementares e suas contradições para a implantação na Estratégia Saúde da Família. In: OLIVEIRA, L. C. F.; NASCIMENTO M. A. A. Práticas integrativas e complementares de saúde no semiárido baiano: utopia ou realidade. Feira de Santana: UEFS Editora; 2017. p.35-84.

FONTENELE, R.P. et al. A Fitoterapia na Atenção Básica: olhares dos gestores e profissionais da Estratégia Saúde da Família de Teresina (PI), Brasil. Ciênc. Saúde Coletiva, Rio de Janeiro, v.18, n.8, p. 2385-2394, 2013.

GIRALDI M.; HANAZAKI N. Uso e conhecimento tradicional de plantas medicinais no Sertão do Ribeirão, Florianópolis, SC, Brasil. Acta Bot. Bras., Belo Horizonte, v. 24, n.2, p.395-406, 2010.

LEITE, S. N.; SCHOR, N., Fitoterapia no Serviço de Saúde: significados para clientes e profissionais de saúde. Saúde em debate, Rio de Janeiro, v. 29, n. 69, p. 78-85, 2005. 
Conhecimento dos agentes comunitários de saúde de um município baiano sobre plantas medicinais

MATSUCHITA, H.L.P.; MATSUCHITA, A.S.P. A Contextualização da Fitoterapia na Saúde Pública. Uniciências, Cuiabá, v.19, n.1, p.86-92, 2015.

MARTINS, A. R.; PEREIRA, D. B.; CEOLIN T. Relato de experiência - o uso de plantas medicinais no cotidiano de trabalho das agentes comunitárias de saúde. Cienc. Cuid.

Saude, Maringá, v.7, 2008. Disponível em:

http://ojs.uem.br/ojs/index.php/CiencCuidSaude/article/view/6721/pdf. Acesso em: 06 jun. 2019.

NICOLETTI, et al. Principais interações no uso de Medicamentos fitoterápicos. Infarma, Brasília, v.19, n. 1/2, p.32-40, 2007.

NICOLETTTI, M. A. et al. Uso popular de medicamentos contendo drogas de origem vegetal e ou plantas medicinais: principais interações decorrentes. Rev. Saúde- UNG online, Guarulhos, v. 4, n. 1, p. 25-39, 2010.

OSUNA, J. T. A. et al. Cultivo, beneficiamento e considerações sobre usos de plantas medicinais. Feira de Santana-Ba: UEFS Editora, 2005.

RANGEL, M.; BRAGANCA, F. C. R. Representações de gestantes sobre o uso de plantas medicinais. Rev. bras. plantas med. Paulínea, v.11, n.1, p.100-109, 2009.

RATES, S.M.K.. Promoção do uso racional de fitoterápicos: uma abordagem no ensino de Farmacognosia. Rev. bras. farmacogn., Maringá, v.11, n. 2, p. 57-69, 2001.

SANTOS, E.C.; ALENCAR, B.R.; PIRES, G.B.; ALENCAR, T.O.S. Uso de plantas medicinais no Brasil uma revisão de literatura de 2009 a 2014. Rev. Bras. Farm., Rio de Janeiro, v. 98, n.1, p. 1876-1892, 2017. Disponível em: http://www.rbfarma.org.br/files/RBFarma---N98---V1---2017.pdf. Acesso em: 06 jun. 2019.

SCHIAVO, M.; SCHWAMBACH, K. H; COLET, C.F. Knowledge on medicinal plants and herbal medicines by community health agents of Ijuí/RS. Rev Fund Care Onlin, v. 9, n. 1, p.57-63, 2017. Disponível em: $<$ http://www.seer.unirio.br/index.php/cuidadofundamental/article/view/4271/pdf $>$. Acesso em: 07 dez. 2019.

SCHULZ, V.; HANSEL, R.; TYLER, V. E. Fitoterapia racional: um guia de fitoterapia para as ciências da saúde. 4. ed. Barueri: Manole, 2002.

SILVA J. P. A et al. Plantas medicinais utilizadas por portadores de diabetes mellitus tipo 2 para provável controle glicêmico no município de Jequié-BA. Rev. Saúde. Com, Jequié, v. 4, n.1, p. 10-18, 2008. Disponível em:

http://periodicos2.uesb.br/index.php/rsc/article/view/116/86. Acesso em: 06 jun. 2019.

VEIGA JUNIOR, V. F.; PINTO, A. C.; MACIEL, M. A. M. Plantas medicinais: cura segura. Quím. nova, São Paulo, v. 28, n. 3, p. 519-528, 2005. 
Conhecimento dos agentes comunitários de saúde de um município baiano sobre plantas medicinais

VEIGA JUNIOR V. F. Estudo do consumo de plantas medicinais na Região Centro-Norte do Estado do Rio de Janeiro: aceitação pelos profissionais de saúde e modo de uso pela população. Rev. bras. Farmacognosia, João Pessoa, v.18, n.2, p. 308-313, 2008.

WHO. Estrategia de la OMS sobre medicina tradicional 2014-2023. Organización Mundial de la Salud, 2013. Disponível em:

https://apps.who.int/medicinedocs/documents/s21201es/s21201es.pdf. Acesso em: 06 jun. 2019.

Recebido em: 11/06/2019

Aceito em: 12/12/2019 